\title{
Ensuring Reliable Communication in Disaster Recovery Operations with Reliable Routing Technique
}

\author{
Varun G. Menon, ${ }^{1}$ Joe Prathap Pathrose, ${ }^{2}$ and Jogi Priya ${ }^{3}$ \\ ${ }^{1}$ Department of Computer Science Engineering, Sathyabama University, Tamil Nadu 600119, India \\ ${ }^{2}$ Department of Information Technology, RMD Engineering College, Chennai 601206, India \\ ${ }^{3}$ Department of Computer Science Engineering, Karpagam University, Coimbatore 641021, India
}

Correspondence should be addressed to Varun G. Menon; varunmenon@scmsgroup.org

Received 1 October 2015; Revised 31 December 2015; Accepted 14 January 2016

Academic Editor: Claudio Agostino Ardagna

Copyright (C) 2016 Varun G. Menon et al. This is an open access article distributed under the Creative Commons Attribution License, which permits unrestricted use, distribution, and reproduction in any medium, provided the original work is properly cited.

\begin{abstract}
The purpose of this research paper is to ensure reliable and continuous communication between the rescue officers and other people during disaster recovery and reconstruction operations. Most of the communication infrastructure gets damaged during the disaster and proper communication cannot be established in the area which leads to longer delays in emergency operations and increased damage to life and property. Various methods proposed to enable communication between the people using wireless ad hoc networks do not guarantee reliable delivery of data with fast moving devices. This paper presents a Reliable Routing Technique (RRT) that ensures reliable data delivery at the destination device even when the people with the mobile devices are moving in the network. We make use of the broadcasting property of the wireless network and create a priority list of probable forwarding candidates at each device. With this technique, RRT ensures that if a forwarder device is unable to forward the data packet due to movement of mobile devices, the next priority candidate forwards the data packet to the destination device, thus ensuring reliability of data delivery in the network. Simulation results show that RRT achieves significant performance improvement with better data delivery in ad hoc networks.
\end{abstract}

\section{Introduction}

The world has witnessed a number of natural disasters over these years, causing huge losses to human and animal life, infrastructure, and almost everything in the region. These natural hazards like earthquakes, floods, and hurricanes have always struck in different places at unpredictable times, leading to the increase in damage of life and property. Although science has made vast progress in many areas, scientists are still unable to accurately predict the time and place of these disasters and the extent of damage that might occur. The Indian Tsunami in $2004[1,2]$ is one among many that have made us realize the extent of damage a natural disaster can cause in unpredictable situations. So the major focus has always been on minimizing the damage that might be caused by a natural disaster and to stay ready for disaster recovery and reconstruction operations [3].
Disaster recovery and reconstruction operations have always been a challenging task for the government, local authorities, and the people. The primary aim of the firemen, police officers, local guards, and other rescue officers arriving just after the event is to look for the survivors and to help the injured. These first responders arriving at the site immediately after the disaster have to deal with a number of issues and challenges. In some cases it is necessary for them to prevent the damage from spreading to other areas. They have to search for the survivors within the damaged buildings and also have to make sure that the medical assistance reaches the survivors in minimum time. Once the survivors are found and medical assistance is given, the next major task is to rebuild the basic infrastructure to start the reconstruction works. A major issue during these recovery and reconstruction operations is that most of the infrastructure used for transportation, communication, and so forth would have been completely or partially damaged 
with the disaster and it becomes quite difficult to handle and coordinate the entire process without the help of this infrastructure.

One of the most important requirements in the disaster recovery and emergency response situations is to establish reliable and continuous communication between the officers, medical team, and other rescue workers [4]. Effective communication is very important in coordinating the rescue work and also in reconstruction works after the disaster. In order to carry out efficient and quick recovery, the rescue workers, police officers, and everyone involved may have to move at a fast pace to different locations within the area to minimize the damage and to find out more survivors of the disaster. But in most of the situations the communication infrastructure gets damaged and proper communication cannot be established in the area which leads to longer delays in emergency operations and increased damage to life and property [5].

A number of solutions have been proposed over these years to establish communication in disaster management services [6-8]. The use of ad hoc networks [9-11] is one of the best techniques used in establishing communication during disaster relief operations. Ad hoc wireless networks are a collection of mobile devices that can be configured to work as a single network and can be deployed in these areas without the help of any infrastructure or centralized control. Any number of mobile devices like mobile phones and personal computers can be attached to the ad hoc network. Every mobile device is free to join or leave the network at any point of time. Every device in the network acts as the router as well as the host. When a mobile device sends a data packet into the network, the device in its transmission range receives the data packet and then forwards the packet to the next device in its transmission range and so on till it reaches the destination. Although a number of techniques have been proposed for the transmission of the data from the source to the destination, due to constant movement of mobile devices, none of the methods guarantee delivery of the information at the destination [12, 13]. Also most of the methods do not support continuous communication between the mobile devices [14]. It is very important for all the people at the place of the disaster including the survivors and rescue workers to communicate with each other while moving from one place to the other at a fast pace for safety to minimize the damage. Thus reliable delivery of information and continuous communication become two important factors in efficient working of disaster recovery operations even with fast moving people using devices like mobile phones, laptops, iPads, and so forth.

This paper provides a new technique called Reliable Routing Technique (RRT) that utilizes opportunistic routing to guarantee the delivery of information at the destination device. We use the term data packet for the information passed in the wireless network. When a device sends a data packet into the network, all the devices in its transmission range receive the data packet. We create a priority list of these mobile devices. The mobile device that is nearest to the destination is given the highest priority and is always selected to forward the data packet to the next device. If that particular mobile device moves away during this time, the next priority mobile device forwards the data packet in the network. Thus communication is maintained, as long as one mobile device receives the transmission and thus the delivery of data packet at the destination is guaranteed. Simulation results show that RRT achieves high data delivery even when the mobile devices are moving rapidly from one place to the other.

The first section of this paper discusses the various research work that has already been carried out in the area of reliable communication in disaster management. The next section analyses the reasons for the failure of communication systems during the disaster recovery process and also analyses the importance of telecommunications in disaster recovery and reconstruction processes based on the data collected from the questionnaires and personal interviews. The next section describes the proposed Reliable Routing Technique for reliable and continuous delivery of information during disaster recovery and reconstruction process. Next section explains the implementation details with results and discussions. We conclude the paper in the last section with discussion to future works and enhancements.

\section{Related Work}

A number of research papers have been published highlighting the effects of natural disasters in various regions around the world. Most of these papers have highlighted the importance of being prepared for emergency and disaster recovery works. Reference [15] has reviewed the effect of the earthquake that hit Kobe, Japan, in 1995. The paper highlights the changes that have taken place over time in the region for effective disaster management. Another book [16] reviews the major natural disasters that have hit various regions of the world in 2011 and discusses the effects of natural disasters on the country and its people. Reference [17] highlights the problems and issues that can occur if people are not well prepared for disaster management. Reference [18] explains the various steps that are required for every region for disaster preparation and management with reference to the island of Hawaii. The paper [19] describes the various steps that need to be implemented for prevention and efficient recovery from natural disasters.

The importance of communication during disaster recovery process has been a major area of study over these years with a number of research papers published highlighting the need for effective means of communication in disaster recovery and reconstruction works. Reference [4] has given a detailed explanation of the need for telecommunication during the disaster recovery process. The paper also highlights the various steps that can be implemented as a preparation to meet the disaster recovery process. Reference [20] proposed a public safety communication system by integrating wireless local area network and the radio. Although this was a new approach in public safety communication, the method had scalability problems and some major issues. Reference [10] gave an ad hoc network for disaster relief operations that was used for communication and in tracing people inside damaged buildings. Although the method had a number of additional functions apart from communication, reliable 
delivery and high performance could not be achieved with moving mobile devices. Reference [21] reviewed the various methods that are available for communication in public safety and disaster management.

Reference [22] gave a rapid emergency deployment mobile communication node that uses several communication technologies to provide multiple communication services in disaster management situations. The system also supported live video streaming and multimedia content sharing along with the traditional communication services. The proposed node included a hybrid power source based on both renewable and nonrenewable energy generators and batteries to provide electric autonomy. A pneumatic telescopic mast is installed to support communication antennas providing mobility and increased coverage range. One or more nodes can be easily and quickly deployed in any location of interest to provide communication services. Reference [23] proposed a flexible network architecture that provided a common networking platform for heterogeneous multioperator networks, for interoperation in case of emergencies. Reference [24] gave excellent techniques using key agreements to enhance the security of mobile wireless networks. Reference [25] discussed the communication services that can be exploited during the disaster recovery and reconstruction operations.

As the focus was more on deploying the ad hoc network and its security, fewer papers have worked on reliable and continuous data delivery between the fast moving mobile devices. The traditional topology based protocols like DSR [26] and DSDV [27] suffer from increased node mobility. These protocols are much more focused on fixed routes and their performance decreases with dynamic topology and node mobility. This leads to data loss in the network which is unacceptable in emergency situations. The idea of geographic routing [28] provided a much reliable and better way of transferring data in ad hoc networks. Geographic routing uses location information or the geographic position of the node to transfer data from one node to the other. The location information is transferred as one-hop beacon between the nodes. GPSR [29] is one of the most popular geographic routing protocols that use greedy forwarding and perimeter routing to transfer data in dynamic wireless networks. But even geographic routing suffers from a major drawback that it is very sensitive to inaccuracies in location information and its performance comes down with high mobility of nodes. This gave way to opportunistic routing and opportunistic forwarding [30]. The broadcasting nature of wireless networks was exploited with the ExOR [30] opportunistic routing protocol. ExOR protocol provided an improved way to utilize the broadcasting property of the wireless links to enhance communication at the data link and network layers of multihop wireless networks that remained static. It is a hybrid routing and MAC protocol for wireless networks that improves the data delivery of unicast transmissions. Here the sender broadcasts a batch of packets. Every packet has a list of nodes which can forward it. To maximize the progress of each transmission, the forwarding node would send data packets in the order of their nearness to the destination node. To reduce redundant transmissions, ExOR uses a batch map which would store the list of packets received at each node; every forwarding node would only forward data that has not been acknowledged by the nodes nearer to the destination in their particular batch maps. ExOR provides significant throughput improvement over earlier routing strategies but ExOR cannot support multiple simultaneous flows and thus limits the practical use of this protocol in these fast reconfiguring networks. This problem was addressed by the SOAR [31] protocol. SOAR supports simultaneous flows in multiple paths. It also incorporates adaptive forwarding path selection to leverage path diversity and minimizes duplicate transmissions. But one of the major limitations with this protocol is that it uses link state style topology database for routing. In order to determine the rate of loss of packets, we often require periodic networkwide updates and measurement. This would be impractical in wireless networks with highly dynamic nodes. In order to avoid this problem some protocols introduced the batching system [32]. But many applications using this system incurred much delay in packet arrival. Most of the recent location based routing schemes [33] do not entirely address the problem of additional memory consumption and overhead incurred. A community aware opportunistic routing [34] was proposed to work on mobile nodes with social characteristics. Reference [35] proposed an opportunistic routing scheme to handle communication voids in fast moving ad hoc networks. Reference [36] proposed a parallel routing scheme which is performed by many nodes simultaneously to maximize the opportunistic gain while controlling the interuser interference. Most of these routing protocols suffered from one or more disadvantages and were not able to guarantee reliable data delivery at the destination. This has motivated us to work on this new method of RRT that guarantees reliable delivery of information between the rescue workers during the time of disaster and in its recovery and reconstruction phases.

\section{Analysing the Reasons for the Failure of Communication Systems during the Time of Disaster}

Data for the analysis was collected through questionnaires from the people affected by 2013 Uttarakhand floods [37] in India. Personal interviews were carried out with the rescue workers and other people who took part in the disaster recovery and reconstruction works. The questionnaires and interviews were focused on two major things: (1) the reasons for the failure of communication systems during the time of disaster and (2) the importance of a reliable communication network during the disaster.

Figure 1 shows the analysis of the data collected through questionnaires. Out of 419 responses received, 321 (76.61\%) people indicated that the primary reason for the failure of telecommunication systems during the disaster is the destruction of communication infrastructure and network elements. $74(17.66 \%)$ people indicated the isolation and failure of supporting elements in communication as the major reason for the failure. 21 (5.01\%) people were of the opinion that the excess network load and network congestion were responsible for the failure of communication during the time 


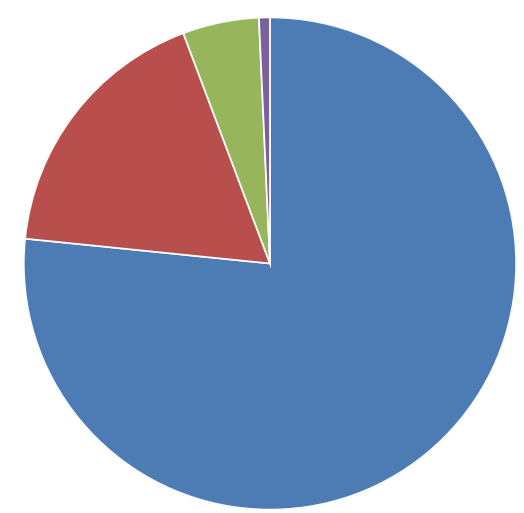

- Destruction of communication infrastructure and network elements

- Isolation and failure of supporting elements in communication

- Network overload and congestion

Unable to answer

FIGURE 1: Reasons for the failure of communications system: data from the questionnaires.

of the disaster and in the recovery process. Figure 2 shows the analysis of the data collected through personal interviews. Out of 44 rescue workers interviewed, 20 (45.45\%) people indicated that the primary reason for the failure of telecommunication systems during the disaster is the destruction of communication infrastructure and network elements, $16(36.36 \%)$ people indicated the isolation and failure of supporting elements in communication as the major reason for the failure, and $8(18.18 \%)$ people were of the opinion that the excess network load and network congestion were responsible for the failure of communication during the time of the disaster and in its recovery process.

From the above results we concluded that the failure of communication systems during natural disaster and in recovery and reconstruction works occurs mainly due to these three reasons with most of the people citing the destruction of physical infrastructure needed for communication as the primary reason.

(i) Destruction of Communication Infrastructure and Network Elements. Natural disaster often destroys the physical communication infrastructure and network components like the transmission towers, base stations, and so forth. Most of these telecommunication equipment pieces like transmission towers are very much prone to natural disasters due to their structure and place of deployment. Once this physical telecommunication infrastructure gets destroyed, it becomes very difficult to have proper means of communication in the disaster affected areas.

(ii) Isolation and Failure of Supporting Elements in Communication. This is one of the major challenges faced in ensuring communication during the time of the disaster and afterwards. The infrastructure like electricity supply, transportation systems, and so forth that supports communication infrastructures gets damaged during these unpredictable events. These supporting elements play a vital role in

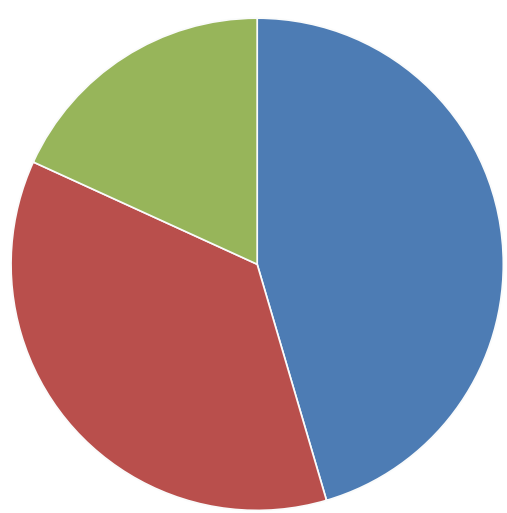

- Destruction of communication infrastructure and network elements - Isolation and failure of supporting elements in communication - Network overload and congestion

FIGURE 2: Reasons for the failure of communications system: data from the interviews.

the telecommunication sector and thus entire communication system is hampered by the destruction of these elements.

(iii) Network Overload and Congestion. During the time of a disaster, most of the people try to communicate with others and overload the available communication bandwidth.

\section{Analysing the Importance of Reliable and Continuous Communication during the Disaster}

Communication networks play a vital role in disaster management services. In this section we analyze the importance of reliable and continuous communication in disaster management services using the data obtained from the questionnaires and interviews. Figure 3 shows the analysis of the data collected through questionnaires. We used Likert Scale to mark the responses. Out of 419 responses received, $345(82.33 \%)$ people indicated that reliable and continuous communication was "very important" during the disaster and in disaster management services. 40 (9.54\%) people called it "important" while 29 (6.9\%) people indicated it as "moderately important." A small minority, 3 (0.007\%) people, called it "of little importance" and $2(0.004 \%)$ people called it "unimportant." Figure 4 shows the analysis of the data collected through personal interviews.

Out of 44 rescue workers interviewed, 38 (86.36\%) people indicated that reliable and continuous communication was "very important" during the disaster and in disaster management services. Four (9.09\%) people indicated that reliable communication was "important," while 2 (4.5\%) people indicated it as "moderately important." None of the rescue workers selected the "of little importance" and "unimportant" options. So it is evident that all the rescue workers that took part in disaster recovery and reconstruction works regard 


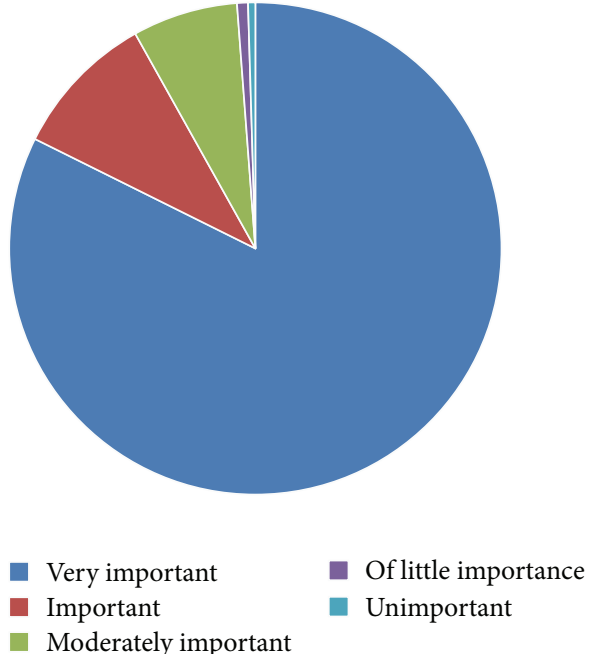

FIGURE 3: Importance of reliable and continuous communication during the disaster: data from questionnaires.

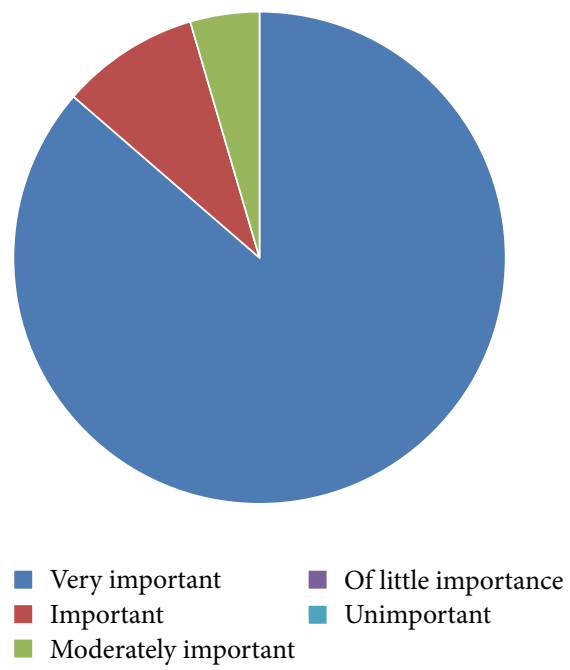

FIGURE 4: Importance of reliable and continuous communication during the disaster: data from interviews.

reliable communication as a very important factor in disaster management.

From the analysis it is very evident that reliable and continuous communication between the people, rescue workers, and everyone at the site was extremely important during the time of the disaster and also afterwards in disaster recovery and reconstruction processes. So it is necessary to provide a technique for reliable and continuous communication between the rescue officers and various people involved during the disaster recovery process to ensure minimum damage to life and property.

\section{Reliable Routing Technique (RRT)}

The working of the Reliable Routing Technique (RRT) is illustrated in Figure 5. The small circles depict the wireless

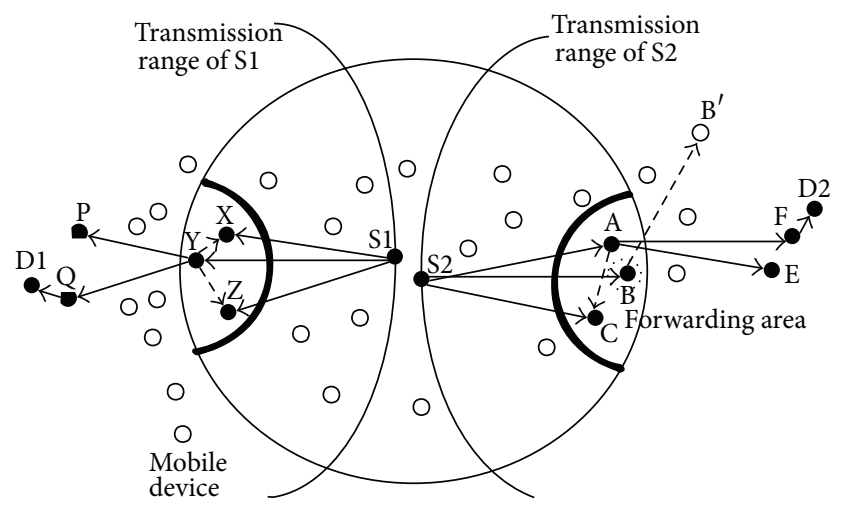

FIGURE 5: Data transferred between the wireless nodes using RRT.

devices like mobile phones, laptops, iPads, and so forth, used by different people in the disaster hit area. Let us first consider device S1 held by a rescue officer trying to send information to device D1 held by another rescue officer in the wireless network. Wireless devices have a unique property of broadcasting every data piece it receives into the network [38]. Wireless devices communicate with each other by broadcasting every data packet it receives into the network. We make use of this property in designing our new method. Using this property of the wireless medium the person using the mobile device $\mathrm{S} 1$ broadcasts the information intended for the person with device D1 into the wireless ad hoc network.

Position of the destination is obtained using a location registration and lookup service used in [29]. This service would map node addresses to the locations. We consider two situations to implement the working of Reliable Routing Technique (RRT). In the first situation we assume that there are no disruptions and problems in the wireless link and wireless channel and there is no movement of the devices out of the transmission area. Mobile devices $\mathrm{X}, \mathrm{Y}$, and $\mathrm{Z}$ which are in device Sl's transmission range receive the data packet. We create a priority list of these devices such that the mobile device that is nearer to the destination is selected as the device which would forward the data further towards the destination device. We then share the priority list between the neighboring devices. So, based on the priority list, device $\mathrm{Y}$ is selected to forward the data packet or the information towards the destination device. Device Y would first check whether the destination device is in its transmission range. If yes, it would directly deliver the data packet to the destination. If not, device $\mathrm{Y}$ broadcasts the data packet towards the destination. Devices $\mathrm{X}$ and $\mathrm{Z}$ which are in the transmission range of $\mathrm{Y}$ also receive a copy of the same data packet and thus they realize that the data packet has been already forwarded by another best forwarder mobile device. So they drop the data packet. Meanwhile the packet is received by devices P and Q. Based on the priority list $\mathrm{P}$ has the maximum progress towards the destination. Mobile device $\mathrm{P}$ would initially check whether the destination device $\mathrm{D} 1$ is in its transmission range or not. As it finds destination D1 in its transmission range, it delivers the data packet to the destination.

In the second scenario, we assume that the rescue officers with mobile devices are moving to different places. Let us 
consider device S2 held by a rescue officer trying to send information to device D2 held by another rescue officer in the wireless network. Device S2 broadcasts the data which is received by $A, B$, and $C$. Based on the method we would select device $B$ as the first priority candidate to forward the packet. But device $B$ moves out of the transmission range because of the movement of the rescue officer handling device $B$. Thus device $B$ is unable to receive the data packet. We have set a timer $(\mathrm{T})$ for every device. Once the timer expires and devices $\mathrm{A}$ and $\mathrm{C}$ do not receive the copy of the same data packet (devices $\mathrm{A}$ and $\mathrm{C}$ have already obtained one copy of the data packet from S2), based on the priority list set, the second priority device, device A forwards the packets to the destination device. Similarly device F receives the data packet and delivers it to the destination device. So as long as there is one device in the priority list, the delivery of the data at the destination device and continuous communication is guaranteed.

5.1. Developing Priority List of Forwarding Candidates. We construct a priority list of forwarding nodes in the network, so that if one node is unable to receive or forward the packet, the next priority node can do it, thus ensuring continuous communication even with highly mobile nodes. We have set the priority list in such a way that only nodes located in the forwarding area would be given priorities and the chance to forward the packet.

Algorithm for constructing the forwarder priority list is as follows:

initialization,

set the destination node as $\mathrm{N}_{\mathrm{D}}$,

set the Forwarder Priority List as FPL and initialize its value to zero,

set the Neighbor Node List as NNL,

set the transmission range as $T_{R}$,

set the distance from the current node to the destination node $\mathrm{N}_{\mathrm{D}}$ as $\mathrm{CN}_{\text {DIST }}$ :

(1) begin

(2) if destination node is in the list of neighbors then

(3) set destination node as the next hop node

(4) return

(5) end if

(6) for $\mathrm{j} \leftarrow 0$ to length(NNL) do

(7) $\mathrm{NNL}[\mathrm{j}] . \operatorname{dist} \leftarrow \operatorname{dist}\left(\mathrm{NNL}[\mathrm{j}], \mathrm{N}_{\mathrm{D}}\right)$

(8) end for

(9) NNL.sort()

(10) next_hop $\leftarrow$ NNL[0]

(11) for $\mathrm{j} \leftarrow 1$ to length(NNL) do

(12) if $\operatorname{dist}\left(\mathrm{NNL}, \mathrm{N}_{\mathrm{D}}\right) \geq$ length of FPL or $\mathrm{CN}_{\mathrm{DIST}}$

(13) then

(14) break

(15) else if $\operatorname{dist}(\mathrm{NNL}[\mathrm{j}], \mathrm{NNL}[0])<\mathrm{T}_{\mathrm{R}} / 2$
(16) then

(17) FPL.add(NNL[j]) and set the priority for each node starting from 1

(18) end if

(19) end for

The source node broadcasts data packet intended for the destination into the network. The proposed algorithm (steps (1)-(5)) initially checks whether the destination node is in the list of neighbors of the node receiving the data packet. If the destination node is found the data packet is delivered. Next we consider all the neighboring nodes of the initial node and calculate the distance (steps (6)-(9)) of each neighboring node with the destination and sort the list. Then we allocate priorities to each node, with the node with the shortest distance to the destination getting the first priority, followed by the next and so on. The first priority node would be selected as the best forwarder node by the neighboring node for that particular data transmission. For each node we constantly check (steps (10)-(17)) whether the distance to the next hop node exceeds half of the transmission range of that node and whether it moves way from the transmission range. If it exceeds the distance we would remove it from our list and update the Forwarder Priority List (FPL).

\section{Results and Discussion}

We study the performance and properties of Reliable Routing Technique through simulations in Network Simulator-2 [39]. We had developed different types of topologies with different number of nodes for evaluating the performance. Using Network Simulator-2 we create an environment similar to a disaster hit area with many wireless devices. The movement of the rescue officers is simulated by moving the mobile devices randomly in the network. Initially we carry out simulation with 100 nodes with a uniformly distributed network topology. The packet size is set at 256 bytes and the transmission range is $250 \mathrm{~m}$. The nodes are distributed over a $1000 \mathrm{~m} \times$ $800 \mathrm{~m}$ rectangular region. The two-ray ground propagation model is used for the simulation. Mobility is introduced in the network with the Random Way Point mobility model. The speed of the nodes is then varied from a minimum of $1 \mathrm{~m} / \mathrm{s}$ to various maximum limits in each topology setup to analyse the performance of the protocol in fast changing MANETs with highly mobile nodes. Constant Bit Rate Traffic is generated between the nodes. The simulation time is set at 1000 seconds. We compare and evaluate the performance of RRT with AODV and GPSR protocol based on three metrics. These three metrics are very crucial in deciding the reliability and performance of a routing protocol, especially with highly dynamic nodes.

(i) Packet Delivery Ratio (PDR). It is one of the most important metrics in deciding the performance of a routing protocol in a network. It is defined as the ratio of data packets received at the destination(s) to the number of data packets sent by the source(s). 
TABLE 1: Performance analysis of RRT.

\begin{tabular}{lccc}
\hline $\begin{array}{l}\text { Speed of the } \\
\text { mobile devices } \\
(\mathrm{m} / \mathrm{s})\end{array}$ & $\begin{array}{c}\text { Packet } \\
\text { delivery ratio }\end{array}$ & $\begin{array}{c}\text { Average delay } \\
(\mathrm{s})\end{array}$ & $\begin{array}{c}\text { Length of } \\
\text { path (hops) }\end{array}$ \\
\hline 0 & 0.9992 & 0.003 & 2.1 \\
2 & 0.9991 & 0.003 & 2.1 \\
4 & 0.9991 & 0.006 & 2.1 \\
6 & 0.9987 & 0.01 & 2.1 \\
8 & 0.9985 & 0.01 & 2.1 \\
10 & 0.9985 & 0.011 & 2.2 \\
\hline
\end{tabular}

(ii) Average End-to-End Delay. It is the average delay in receiving an acknowledgement for a delivered data packet.

(iii) Length of Every Path. It is the average end-to-end (node to node) path length for successful packet delivery.

(iv) Data Forwarding Times for Each Hop. It is average number of times a packet is forwarded from network layer to deliver data over each hop.

(v) Data Forwarding Times for Each Packet. It is the average number of times a packet is forwarded from the network layer to deliver the data.

Initially we varied the speed of the mobile devices from 0 to $10 \mathrm{~m} / \mathrm{s}$ and the corresponding values for the performance metrics were noted. Table 1 shows the values of the three performance metrics with varying mobility of the nodes. As the speed of wireless nodes increases there is a small decline in the Packet Delivery Fraction and a small increase in the average delay experienced by the data packet. But as the speed variation is limited to $10 \mathrm{~m} / \mathrm{s}$, much variation in performance metrics cannot be observed. This is because the proposed opportunistic routing scheme works equally well in normal and dynamic scenarios. As a result, even the number of hops taken by the data packet to reach the destination remains the same with a value of 2.1. Further when we increase the speed of nodes to $50 \mathrm{~m} / \mathrm{s}$, more variation is found which is shown in Figures 6-10.

From Figure 6 we can see that using Reliable Routing Technique the Packet Delivery Fraction is very close to 1. As the Packet Delivery Fraction is very close to the optimal value we can interpret that the packet loss that occurred is minimal. This is because RRT guarantees delivery at the destination as long as there is one node in the Forwarder Priority List.

Figures 6, 7, 8, 9, and 10 show the comparison of the performance of RRT with GPSR and AODV protocols. From Figure 6 it is evident that the Packet Delivery Fraction (PDF) of RRT is much better compared to the other two protocols. This implies that the number of packets received at the destination is much larger for the RRT protocol. The PDF value for RRT protocol is very near to 1 , which means that almost all the data packets sent are delivered at the receiver. As the mobility of nodes increases the PDF of GPSR and AODV protocol comes down considerably, but RRT maintains a high delivery ratio. Figure 7 shows that the average delay

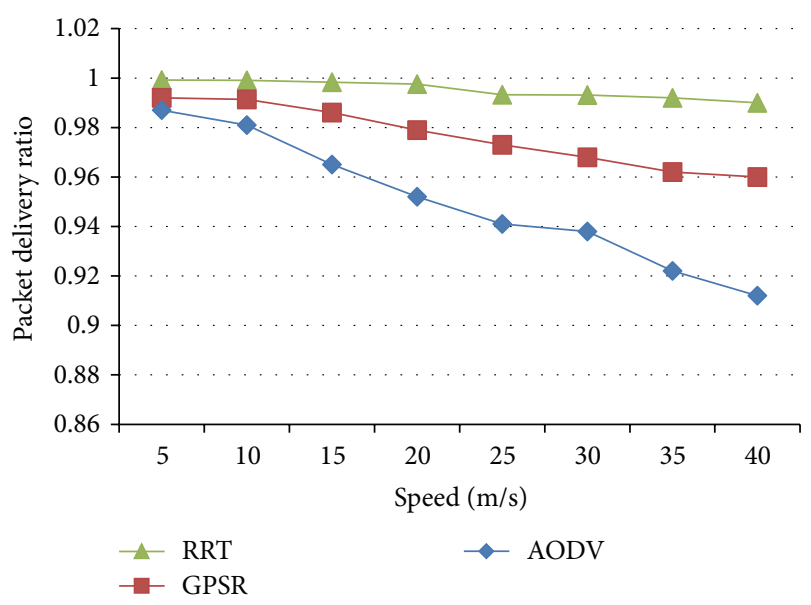

FIGURE 6: Packet delivery ratio versus speed.

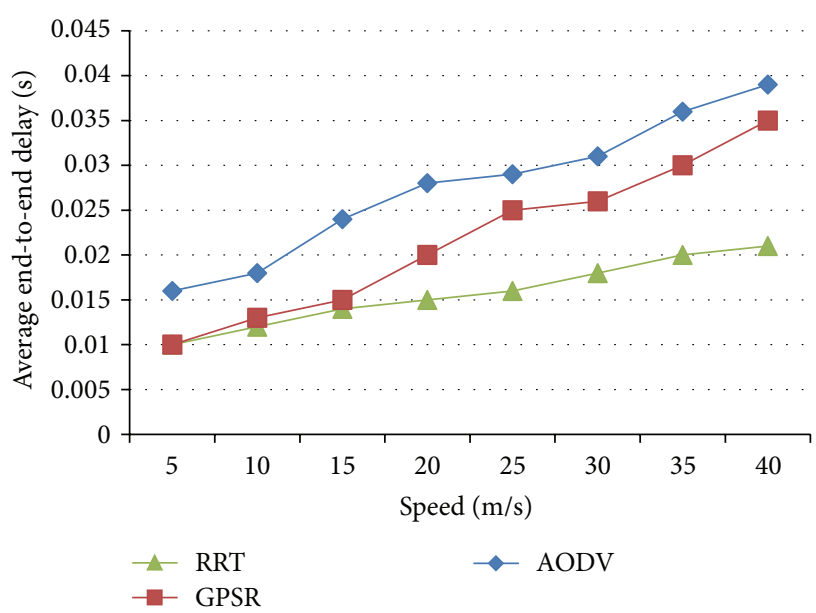

Figure 7: Average delay (source to destination).

experienced by the data packets using RRT protocol is much less compared to the other two. Also as the mobility of the nodes increases, the performance of the GPSR and AODV comes down. Similarly from Figure 8 we can see that RRT takes smaller number of hops to deliver the data packet compared to the other two protocols. These results show that RRT would ensure reliable delivery of data packets with minimum delay in fast changing ad hoc networks.

Figure 9 shows the average packet forwarding times per hop for the three protocols. We can see that the average time taken by RRT to forward a data packet is less compared to GPSR and AODV protocols. This shows that delay experienced by RRT protocol at each hop is very less compared to the other two protocols, leading to RRT's high efficiency. Figure 10 shows the average packet forwarding times per packet for the three protocols. From the figure we can see that RRT takes less time to forward the data packet compared to the other two protocols. This shows that the delay experienced by each packet using RRT is very less compared to the other two protocols in the network. Also we can see that as the speed of nodes increases, the performance of GPSR and AODV comes down but RRT maintains a steady 


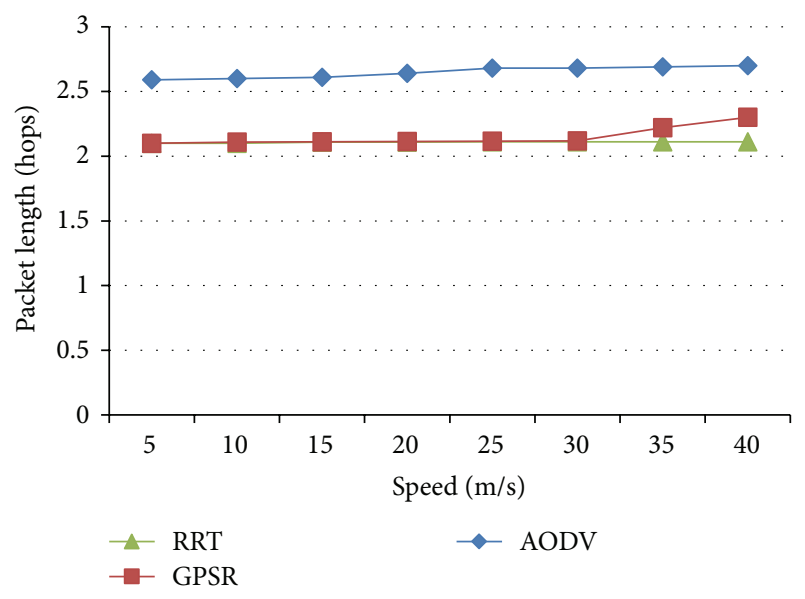

FIGURE 8: Length of every path.

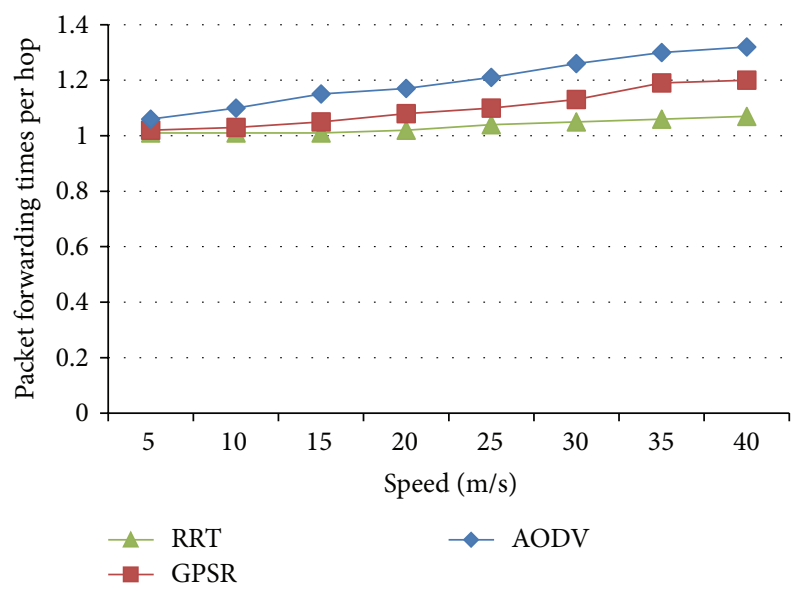

Figure 9: Data forwarding times for each hop.

performance. This shows that the RRT protocol maintains a very good performance even with highly mobile and random nodes in the network. From the simulation results it is evident that RRT achieves better performance compared to the other two protocols and ensures high rate of data delivery even in fast changing and reconfiguring mobile ad hoc networks with highly mobile nodes.

\section{Conclusions}

In this paper we initially analyzed the importance of reliable and continuous communication in disaster recovery and reconstruction works. The data obtained from the questionnaires and personal interviews confirmed that reliable and continuous communication was very important in disaster management services. We also discussed a number of methods given by various researchers for communication in disaster environments. Most of these methods could not guarantee reliable data delivery at the destination device. Using the broadcast property of the wireless medium the proposed Reliable Routing Technique was used for data delivery between two mobile devices in highly mobile ad hoc

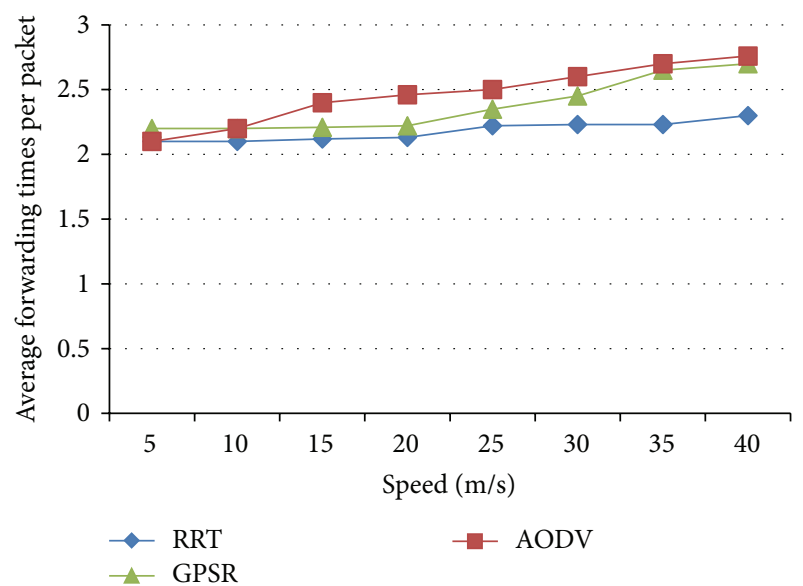

FIGURE 10: Data forwarding times for each packet.

networks. Results from the simulations and comparisons with the other popular data transfer methods confirmed that our method gave very high performance and guaranteed reliable data delivery at the destination device and also ensured continuous communication between the devices.

\section{Conflict of Interests}

The authors declare that there is no conflict of interests regarding the publication of this paper.

\section{References}

[1] R. Shaw, "Indian ocean tsunami and aftermath: need for environment-disaster synergy in the reconstruction process," Disaster Prevention and Management, vol. 15, no. 1, pp. 5-20, 2006.

[2] M. P. Escaleras and C. A. Register, "Mitigating natural disasters through collective action: the effectiveness of Tsunami early warnings," Southern Economic Journal, vol. 74, no. 4, pp. 10171034, 2008.

[3] K. Lorincz, D. J. Malan, T. R. F. Fulford-Jones et al., "Sensor networks for emergency response: challenges and opportunities," IEEE Pervasive Computing, vol. 3, no. 4, pp. 16-23, 2005.

[4] A. M. Townsend and M. L. Moss, Telecommunications Infrastructure in Disasters: Preparing Cities for Crisis Communications, Center for Catastrophe Preparedness and Response, Robert F. Wagner Graduate School of Public Service, New York University, New York, NY, USA, 2005.

[5] R. E. Krock, "Lack of emergency recovery planning is a disaster waiting to happen," IEEE Communications Magazine, vol. 49, no. 1, pp. 48-51, 2011.

[6] Y.-M. Lee, B.-L. Ku, and D.-S. Ahn, "A satellite core network system for emergency management and disaster recovery," in Proceedings of the International Conference on Information and Communication Technology Convergence (ICTC '10), pp. 549552, Jeju, Republic of Korea, November 2010.

[7] K. Mase, N. Azuma, and H. Okada, "Development of an emergency communication system for evacuees of shelters," in Proceedings of the IEEE International Wireless Communications and Networking Conference (WCNC '10), pp. 1-6, Sydney, Australia, April 2010. 
[8] K. Mase, "How to deliver your message from/to a disaster area," IEEE Communications Magazine, vol. 49, no. 1, pp. 52-57, 2011.

[9] I. Chlamtac, M. Conti, and J. J.-N. Liu, "Mobile ad hoc networking: imperatives and challenges," Ad Hoc Networks, vol. 1, no. 1, pp. 13-64, 2003.

[10] N. Pogkas, G. E. Karastergios, C. P. Antonopoulos, S. Koubias, and G. Papadopoulos, "Architecture design and implementation of an ad-hoc network for disaster relief operations," IEEE Transactions on Industrial Informatics, vol. 3, no. 1, pp. 63-72, 2007.

[11] C.-C. Shen, C. Srisathapornphat, and C. Jaikaeo, "An adaptive management architecture for ad hoc networks," IEEE Communications Magazine, vol. 41, no. 2, pp. 108-115, 2003.

[12] E. M. Royer and C.-K. Toh, "A review of current routing protocols for ad hoc mobile wireless networks," IEEE Personal Communications, vol. 6, no. 2, pp. 46-55, 1999.

[13] V. G. Menon and P. M. Joe Prathap, "Performance analysis of geographic routing protocols in highly Mobile Ad Hoc network," Journal of Theoretical and Applied Information Technology, vol. 54, no. 1, pp. 127-133, 2013.

[14] V. G. Menon and P. M. Joe Prathap, "Performance of various routing protocols in mobile ad hoc networks-a survey," Research Journal of Applied Sciences, Engineering and Technology, vol. 6, no. 22, pp. 4181-4185, 2013.

[15] R. Shaw and K. Goda, "From disaster to sustainable civil society: the Kobe experience," Disasters, vol. 28, no. 1, pp. 16-40, 2004.

[16] E. Ferris and D. Petz, The Year that Shook the Rich: A Review of Natural Disasters in 2011, Brookings-Bern Project on Internal Displacement, London, UK, 2012.

[17] P. Rautela and R. K. Pande, "Implications of ignoring the old disaster management plans: lessons learnt from the Amparav tragedy of 23 September 2004 in the Nainital district of Uttaranchal (India)," Disaster Prevention and Management, vol. 14, no. 3, pp. 388-394, 2005.

[18] R. Prizzia, "Coordinating disaster prevention and management in Hawaii," Disaster Prevention and Management, vol. 15, no. 2, pp. 275-285, 2006.

[19] M. Oral, A. Yenel, E. Oral, N. Aydin, and N. Aydin, "Earthquake experience and preparedness in Turkey," Disaster Prevention and Management, vol. 24, no. 1, pp. 21-37, 2015.

[20] A. K. Salkintzis, "Evolving public safety communication systems by integrating WLAN and TETRA networks," IEEE Communications Magazine, vol. 44, no. 1, pp. 38-46, 2006.

[21] G. Baldini, S. Karanasios, D. Allen, and F. Vergari, "Survey of wireless communication technologies for public safety," IEEE Communications Surveys \& Tutorials, vol. 16, no. 2, pp. 619-641, 2014.

[22] I. G. Askoxylakis, A. Makrogiannakis, A. Miaoudakis et al., "A rapid emergency deployment mobile communication node," in Proceedings of the IEEE 19th International Workshop on Computer Aided Modeling and Design of Communication Links and Networks (CAMAD '14), pp. 290-294, Athens, Greece, December 2014.

[23] A. G. Fragkiadakis, I. G. Askoxylakis, E. Z. Tragos, and C. V. Verikoukis, "Ubiquitous robust communications for emergency response using multi-operator heterogeneous networks," EURASIP Journal on Wireless Communications and Networking, vol. 2011, article 13, 2011.

[24] I. G. Askoxylakis, T. Tryfonas, J. May, V. Siris, and A. Traganitis, "A family of key agreement mechanisms for mission critical communications for secure mobile ad hoc and wireless mesh internetworking," EURASIP Journal on Wireless Communications and Networking, vol. 2011, Article ID 807684, 2011.

[25] A. I. Miaoudakis, N. E. Petroulakis, D. Kastanis, and I. G. Askoxylakis, "Communications in emergency and crisis situations," in Distributed, Ambient, and Pervasive Interactions: Second International Conference, DAPI 2014, Held as Part of HCI Interational 2014, Heraklion, Crete, Greece, June 22-27, 2014. Proceedings, vol. 8530 of Lecture Notes in Computer Science, pp. 555-565, Springer, Berlin, Germany, 2014.

[26] D. B. Johnson, D. A. Maltz, and J. Broch, "DSR: the dynamic source routing protocol for multihop wireless ad hoc networks," in Ad Hoc Networking, pp. 139-172, Addison-Wesley, Boston, Mass, USA, 2001.

[27] C. E. Perkins and P. Bhagwat, "Highly dynamic destinationsequenced distance-vector routing (DSDV) for mobile computers," in Proceedings of the Conference on Communications Architectures, Protocols and Applications (SIGCOMM '94), pp. 234-244, ACM, London, UK, 1994.

[28] F. Cadger, K. Curran, J. Santos, and S. Moffett, "A survey of geographical routing in wireless Ad-Hoc networks," IEEE Communications Surveys \& Tutorials, vol. 15, no. 2, pp. 621-653, 2013.

[29] B. Karp and H. T. Kung, "GPSR: greedy perimeter stateless routing for wireless networks," in Proceedings of the 6th ACM Annual International Conference on Mobile Computing and Networking (MobiCom '00), pp. 243-254, Boston, Mass, USA, August 2000.

[30] S. Biswas and R. Morris, "ExOR: opportunistic multi-hop routing for wireless networks," in Proceedings of the Conference on Applications, Technologies, Architectures, and Protocols for Computer Communications (SIGCOMM '05), pp. 133-144, ACM, Philadelphia, Pa, USA, August 2005.

[31] E. Rozner, J. Seshadri, Y. Mehta, and L. Qiu, "SOAR: simple opportunistic adaptive routing protocol for wireless mesh networks," IEEE Transactions on Mobile Computing, vol. 8, no. 12, pp. 1622-1635, 2009.

[32] A. Balasubramanian, R. Mahajan, A. Venkataramani, B. N. Levine, and J. Zahorjan, "Interactive WiFi connectivity for moving vehicles," in Proceedings of the ACM SIGCOMM Conference on Data Communication (SIGCOMM '08), pp. 427-438, ACM, Seattle, Wash, USA, August 2008.

[33] K. Zeng, Z. Yang, and W. Lou, "Location-aided opportunistic forwarding in multirate and multihop wireless networks," IEEE Transactions on Vehicular Technology, vol. 58, no. 6, pp. 30323040, 2009.

[34] M. Xiao, J. Wu, and L. Huang, "Community-aware opportunistic routing in mobile social networks," IEEE Transactions on Computers, vol. 63, no. 7, pp. 1682-1695, 2014.

[35] V. G. Menon and P. M. Joe Prathap, "Opportunistic routing with virtual coordinates to handle communication voids in mobile ad hoc networks," in Advances in Signal Processing and Intelligent Recognition Systems, vol. 425 of Advances in Intelligent Systems and Computing, pp. 323-334, Springer International, 2016.

[36] W.-Y. Shin, S.-Y. Chung, and Y. H. Lee, "Parallel opportunistic routing in wireless networks," IEEE Transactions on Information Theory, vol. 59, no. 10, pp. 6290-6300, 2013.

[37] G. Kabra and A. Ramesh, "An empirical investigation of the enablers in humanitarian supply chain management in India: a case study," Journal of Advances in Management Research, vol. 12, no. 1, pp. 30-42, 2015. 
[38] M. Nikolov and Z. J. Haas, "Towards optimal broadcast in wireless networks," IEEE Transactions on Mobile Computing, vol. 14, no. 7, pp. 1530-1544, 2015.

[39] “The Network Simulator -ns-2," 2013, http://www.isi.edu/nsn$\mathrm{am} / \mathrm{ns} /$. 

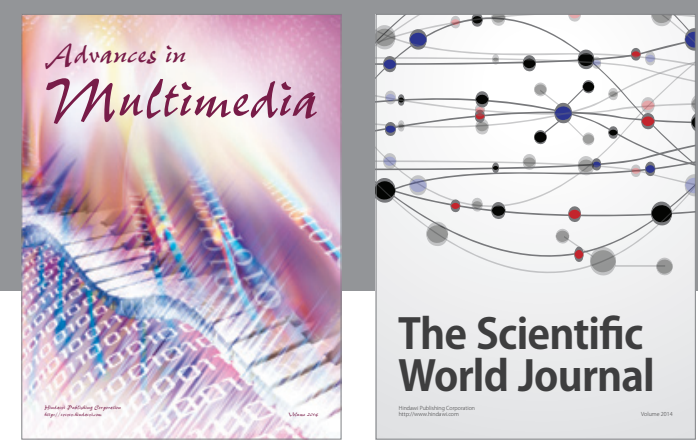

The Scientific World Journal
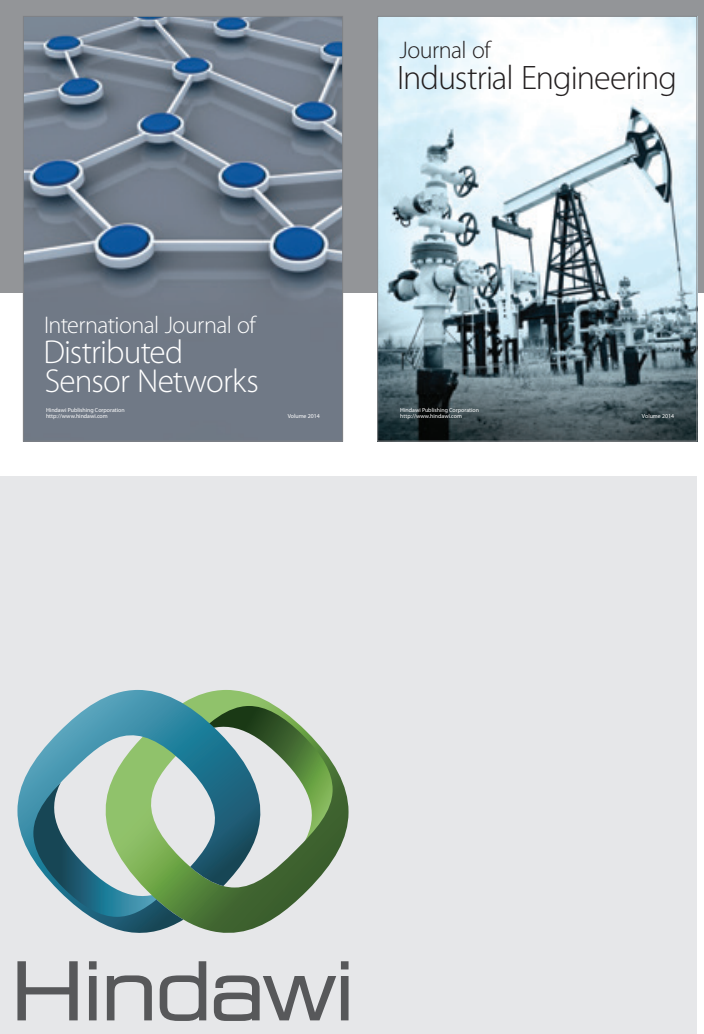

Submit your manuscripts at

http://www.hindawi.com

\section{Computer Networks} and Communications
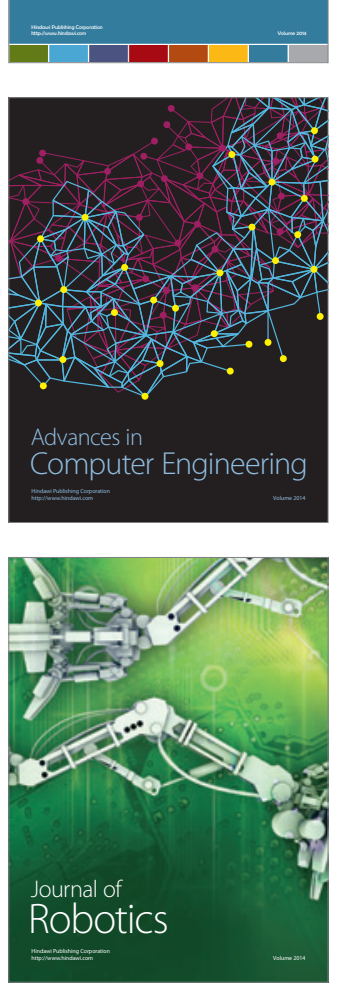
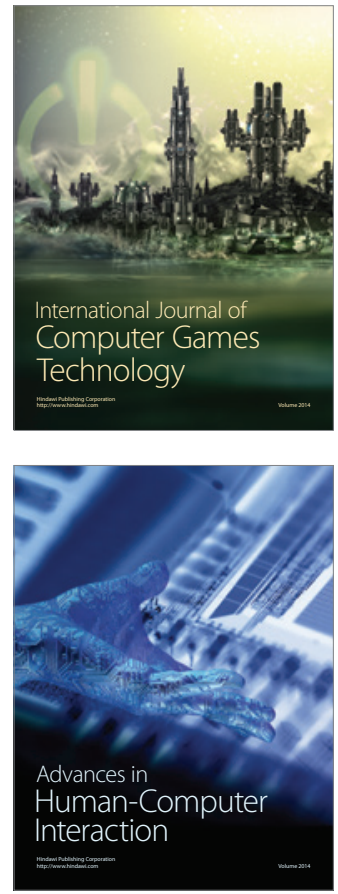
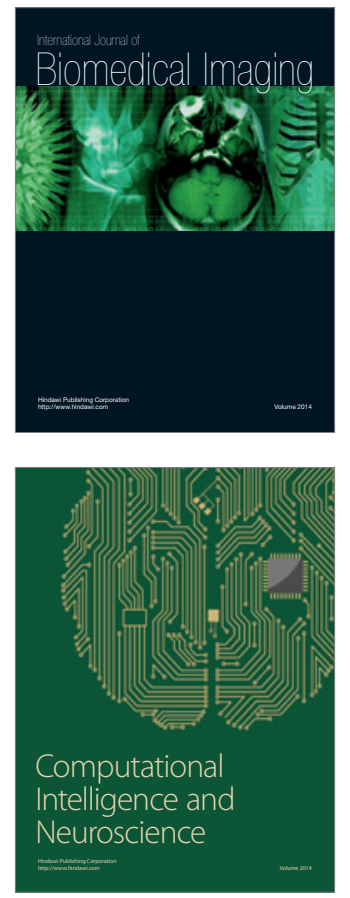
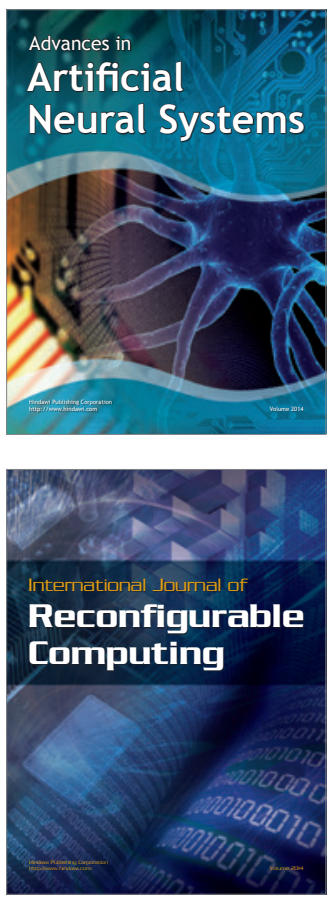
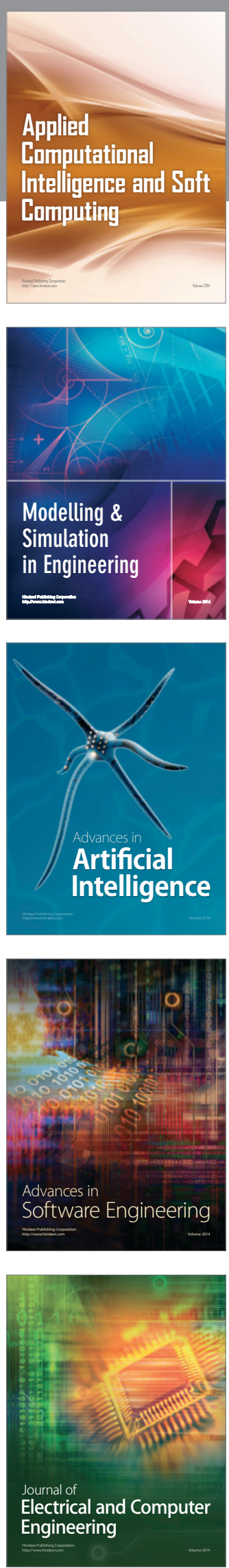